\title{
A new complication of transesophageal echocardiography: Pulmonary embolization of a right atrial myxoma
}

Ashraf Abdelaziz, MD, ${ }^{a}$ Ahmed Abdelgawad, MD, ${ }^{a}$ Mahmoud M. Ramadan, MD, PhD, ${ }^{b}$ and Mohammed Osama, MD, ${ }^{\mathrm{c}}$ Giza and Mansoura City, Egypt

See related commentary page e 82 .

Video clip is available online.

Myxomas form approximately $50 \%$ of benign cardiac tumors, and most of them are located in the left atrium. ${ }^{1}$ When they are in the right atrium, the rare complication

\footnotetext{
From the Department of Cardiothoracic Surgery, ${ }^{\mathrm{a}}$ National Heart Institute, Ministry of Health, Imbaba, Giza; Department of Cardiology, ${ }^{\mathrm{b}}$ Faculty of Medicine, Mansoura University, Mansoura City; and Department of Cardiology, ${ }^{\mathrm{c}}$ National Heart Institute, Ministry of Health, Imbaba, Giza, Egypt.

Disclosures: Authors have nothing to disclose with regard to commercial support.

Received for publication Nov 12, 2014; revisions received Jan 1, 2015; accepted for publication Jan 15, 2015; available ahead of print Feb 20, 2015.

Address for reprints: Mahmoud M. Ramadan, MD, PhD, Department of Cardiology, Specialized Medicine Hospital, Mansoura University, Mansoura City, Egypt

(E-mail: amamod2020@yahoo.com).

J Thorac Cardiovasc Surg 2015;149:e79-81

$0022-5223 / \$ 36.00$

Copyright (C 2015 by The American Association for Thoracic Surgery

http://dx.doi.org/10.1016/j.jtcvs.2015.01.027
}

of massive pulmonary embolism may develop. Treating such cases invariably includes total extraction of the pulmonary embolus, with removal of any remnant of myxoma tissue, to avoid recurrence.

\section{CASE REPORT}

A 43-year-old woman, otherwise healthy, with no medical or surgical history of note, presented for an examination having had a sudden episode of moderate dyspnea 1 month earlier. Physical examination was unremarkable. Chest radiograph was normal, and an electrocardiogram showed a sinus rhythm with P-pulmonale in lead II (Figure 1). Transthoracic echocardiography revealed a mobile, right atrial mass with a long pedicle, protruding through the tricuspid valve (TV) in diastole.

Preoperative transesophageal echocardiography (TEE) was done, to better evaluate the right atrial mass and cardiac valves; and to assess the possibility of the presence of other cardiac masses or congenital septal (and other) defects that may have been missed during transthoracic echocardiography. The patient had fasted for 6 hours before the TEE procedure; the pharynx was anesthetized with a topical anesthetic (lidocaine) spray, applied with the patient in a sitting position. Blood pressure and heart

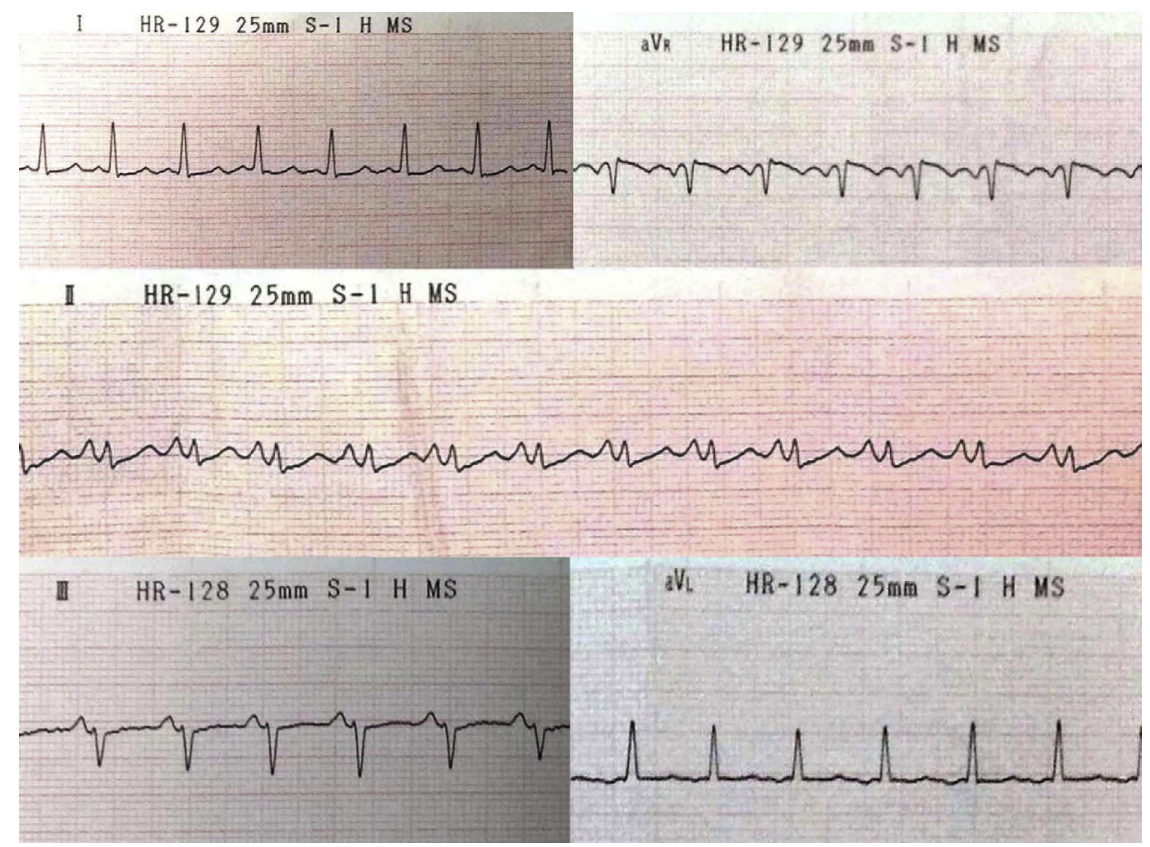

FIGURE 1. Electrocardiogram showing sinus rhythm with P-pulmonale in lead II. 


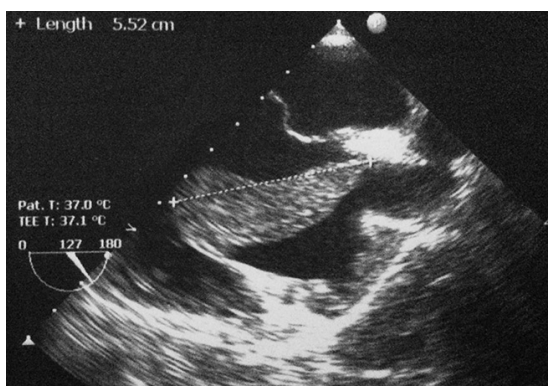

FIGURE 2. TEE showing a large $(5.5 \times 2.5 \mathrm{~cm})$, highly mobile, pearshaped mass inside the right atrium, attached with a narrow pedicle to the superior aspect of the interatrial septum, near the cavoatrial junction of the superior vena cava. Pat., Patient; T, temperature; TEE, transesophageal echocardiography.

rate were continuously monitored during the TEE procedure. The patient was placed in the left-lateral position, and her neck was flexed, to allow for better oropharyngeal entry; a bite guard was used to allow manipulation and protection of the TEE probe, which was adequately lubricated with jelly.

The TEE confirmed the presence of a large $(5.5 \times 2.5$ $\mathrm{cm}$ ), highly mobile, pear-shaped, right atrial mass (Figure 2), attached with a narrow pedicle to the superior aspect of the interatrial septum near the cavoatrial junction of the superior vena cava. To this point, the patient was hemodynamically stable. When the TEE session was nearly completed (Figure 3), the right atrium was suddenly seen to be free from the mass (Figure 4), and the patient developed progressive dyspnea and mild hypotension.

The procedure was abruptly terminated, and a fast screening by transthoracic echocardiography revealed the presence of the right atrial mass in the distal part of the main pulmonary artery, causing its partial obstruction, and total occlusion of the left pulmonary artery, with absent distal flow (Figure 5). This obstruction was associated with a dramatic rise of pulmonary artery systolic pressure, from $40 \mathrm{~mm} \mathrm{Hg}$ before the procedure, to $80 \mathrm{~mm} \mathrm{Hg}$, with severe associated TV regurgitation. The patient was promptly transferred to the operation theater for emergency pulmonary embolectomy.

The surgical approach was via a medial sternotomy with aorto-bicaval cannulation. The superior vena cava was cannulated directly, near the innominate veins, to allow for

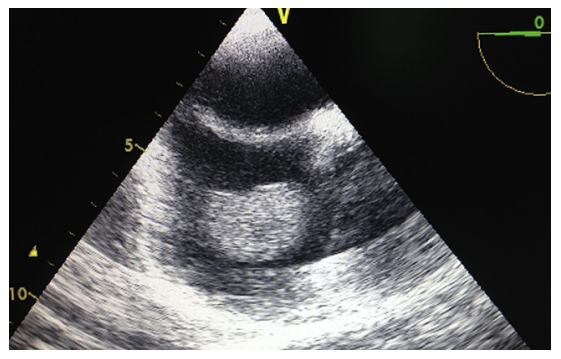

FIGURE 3. TEE showing the mass in the right atrium.

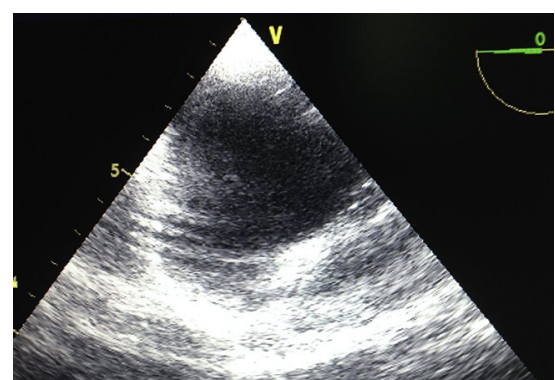

FIGURE 4. The same TEE view showing the disappearance of the mass from the right atrium.

good inspection of the orifice of the cavoatrial junction. Cardiopulmonary bypass was initiated, and the assistedbeating heart technique was chosen. The right atrium was opened parallel to the interatrial groove where the stalk of the embolized mass was seen near the orifice of the cavoatrial junction, but inspection of the right ventricle through the TV revealed nothing abnormal.

The main pulmonary artery was incised longitudinally, just distal to the pulmonary valve annulus, and the incision was extended to the confluence, where the mass was found and caught, using a Duval non-crushing clamp, and gently extracted from the main pulmonary artery (Figures 6 and 7, Video 1). The pedicle of the right atrial mass was totally excised by diathermy, without the need to patch or reconstruct the superior vena cava. A thorough wash of the right atrium and pulmonary artery was done to get rid of any residual masses or other fragments.

While occluding the pulmonary valve, the TV was tested with warm saline before closure of both the right atrium and the pulmonary artery, to exclude any TV complications that may have been caused by the dislodged right atrial mass passing through it. Intraoperative TEE confirmed normal TV function, reduced pulmonary artery pressure, and absent residual masses. The total cardiopulmonary bypass time was 35 minutes; the postoperative period was uneventful; and extubation was done in the first day. On the third postoperative day, the patient was discharged from the intensive-care unit. The histopathologic examination confirmed the tumor to be

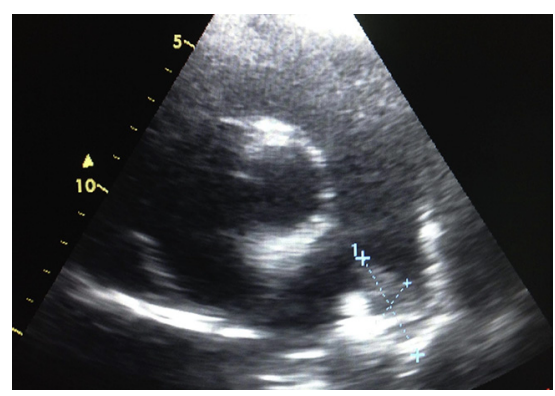

FIGURE 5. Transthoracic echocardiogram (TTE) done immediately after the abrupt termination of TEE session, showing the right atrial mass impacted into the left pulmonary artery. 


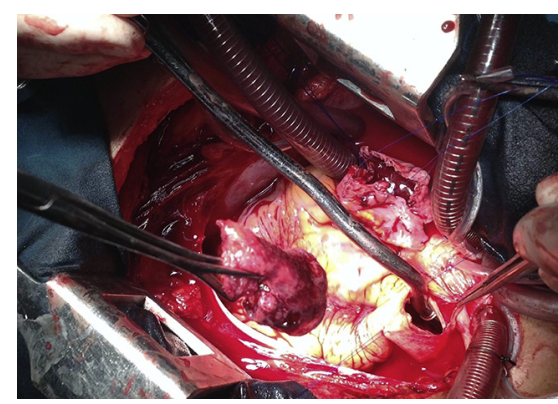

FIGURE 6. The right atrial myxoma being extracted from the right pulmonary artery.

a myxoma. The patient gave consent for her anonymous clinical data to be published in this report.

\section{DISCUSSION}

Pulmonary embolism from right atrial myxoma is a rare event, and the complete removal of both the atrial and pulmonary tumors is of extreme importance to avoid complications and resolve symptoms. ${ }^{2}$ The TEE is a semi-invasive diagnostic technique widely considered to be very useful and superior to transthoracic echocardiography in the preoperative evaluation of cardiac masses, with a very low incidence of complications. ${ }^{3}$ The complications of the TEE procedure described in the literature so far include esophageal perforation, laryngospasm, arrhythmias, hypoxia, and hypotension. ${ }^{4}$ We present here a potential new complication: the first report of a TEE-related embolization of a right atrial myxoma.

The detachment of the right atrial myxoma in this case was clearly temporally related to the TEE procedure, but with no obvious evidence of causation. The right atrial myxoma may have been detached from its pedicle by the esophageal manipulations of the TEE probe. However, as the right atrium is usually far from the esophagus, this patient's anatomy may be aberrant in having the esophagus next to the right atrium (we do not have computed tomographic or magnetic-resonance images to support this possibility). Alternatively, the elevation of right cardiac pressures during an inadvertent Valsalva maneuver (eg, coughing or gagging during the TEE procedure) might have provoked interatrial septum stretching and contributed to the dislodgement of the myxoma.

In fact, the literature includes only 1 report of a fatal pulmonary embolization of a right atrial mass, an event presumably related to the TEE procedure, ${ }^{5}$ but the nature of the embolized mass was not verified (it may have been a thrombus), as the patient died, and a request for consent for a postmortem examination was denied. The delay in the surgical management of that reported case was the main cause of death, as the complication occurred in another hospital where cardiothoracic surgical backup

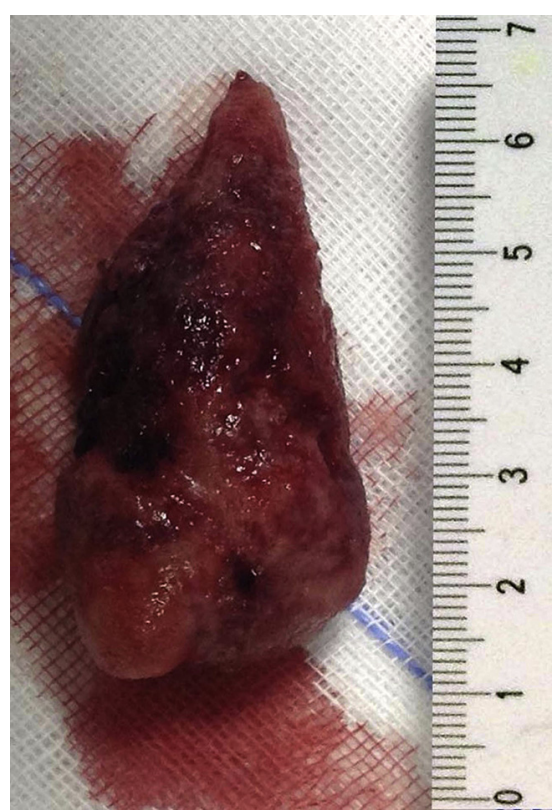

FIGURE 7. Gross appearance of the extracted myxoma, and its actual dimensions.

was unavailable. In the current reported case, the patient survived, owing to both accurate anticipation on the part of the echocardiographer and the urgent transfer to the readily available stand-by cardiothoracic surgical backup team.

This report indicates that preoperative TEE should be avoided in the setting of a mobile, intracardiac mass, particularly when the TEE findings will not change the plan to operate, and especially when TEE will be performed in the operating theater anyway. In addition, when TEE is being done, probe manipulation should be minimized, to identify only the important information, and should be performed by an experienced operator (rather than 1 or multiple Fellows in training), because any left- or right-sided cardiac mass that arises from a narrow stalk is vulnerable to embolization during TEE. Nevertheless, the complication occurred in this case, even though a single, highly experienced operator was involved.

\section{References}

1. Reardon MJ, Smythe WR. Cardiac neoplasms. In: Cohn LH, Edmunds LH Jr, eds Cardiac Surgery in the Adult. 2nd ed. New York: McGraw Hill; 2003:1374-400.

2. Heck HA Jr, Gross CM, Houghton JL. Long-term severe pulmonary hypertension associated with right atrial myxoma. Chest. 1992;102:301-3.

3. Leibowitz G, Keller NM, Daniel WG, Freedberg RS, Tunick PA, Stottmeister C et al. Transesophageal versus transthoracic echocardiography in the evaluation of right atrial tumors. Am Heart J. 1995;130:1224-7.

4. Venkatesh B, Vannan MA, Roelandt JRTC, Pandian NG. Transesophageal echocardiography: laboratory set up, patient preparation and procedure Implementation. In: Roelandt JRTC, Pandian NG, eds. Multiplane Transesophageal Echocardiography. New York: Churchill Livingstone; 1996:15-25.

5. Cavero MA, Cristóbal C, González M, Gallego JC, Oteo JF, Artaza M. Fatal pulmonary embolization of a right atrial mass during transesophageal echocardiography. J Am Soc Echocardiogr. 1998;11:397-8. 